\title{
Particle production and spectroscopy at HERA
}

\author{
A. Falkiewicz ${ }^{\text {a }}$ \\ anP PAN, Kraków, ul. Radzikowskiego 152, Poland
}

Recent results on production of neutral strange $\left(K_{s}^{0}, \Lambda, \bar{\Lambda}\right)$, charged and exotic $\left(\Theta^{+}, \Theta_{c}\right)$ hadrons in ep collisions at HERA are presented. Measurements are compared with various Monte Carlo calculations.

\section{INTRODUCTION}

Recent results on the properties of the hadronic final states in Deep Inelastic Scattering (DIS) are reviewed. The date samples were collected by $\mathrm{H} 1$ and ZEUS collaborations at the $e p$ collider HERA at a centre of mass energy $300-320 \mathrm{GeV}$.

\section{INCLUSIVE CHARGED PARTICLE SPECTRA}

Recently the H1 Collaboration has measured the cross section normalised scaled momentum distribution $\left(x_{p}\right)^{1}$ in the current region of Breit frame $^{2}$ in DIS at high $Q^{2}\left(Q^{2}>100 G e V^{2}\right)[1]$. Compared with previous results [2-7] presented by HERA experiments this measurement extends to higher $Q^{2}$ and to the full range of $x_{p}$.

The energy scale for the current region, set by the virtual photon $(Q / 2)$ is equivalent to one half of the $e^{+} e^{-}$centre of mass energy $E^{\star} / 2$ corresponding to one hemisphere.

The cross section normalised scaled momentum distribution of charged final state hadrons as a function of $Q$ for nine different intervals of $x_{p}$

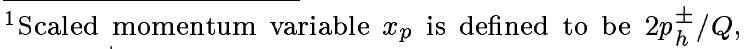
where $p_{h}^{ \pm}$is the momentum of charged track in the current region of the Breit frame.

${ }^{2}$ In the Breit frame and within the quark-parton model (QPM)the purely space-like virtual photon has longitudinal momentum $-Q$, where $Q$ is the virtuality of the exchange boson, and collides elasticaly and head on with (massless) quark of longitudinal momentum $Q / 2$. The struck quark is scattered with an equal but opposite momentum while the proton remnant fragments into the opposite hemisphere. Hadrons emerging from the interaction with negative longitudinal momenta are assigned to the current region.
}

is shown in Figure 1. Also shown is comparison to results from various $e^{+} e^{-}$experiments[15-18]. The $e^{+} e^{-}$data show the same behaviour as the $e p$ data. The agreement confirms the quark fragmentation universality in $e p$ collisions and $e^{+} e^{-}$ annihilation. At lower $Q$ this universality is observed to break down due to higher order QCD processes such as Boson Gluon Fusion (BGF) and Initial state Compton QCD (which can occur before the hard interaction in $e p$ scattering but not in $e^{+} e^{-}$annihilation).

The distributions are compared to different models of the fragmentation process (parton showers (PS)[8], soft color interactions (SCI) [9] and the color dipole model (CDM)) and of the hadronisation process (string[10] and cluster[11]) used in leading order (LO) matrix element Monte Carlo (MC) programs (RAPGAP[12], DJANGO[13], HERWIG[14]), which have been tuned to $e^{+} e^{-}$results. Figure 2 shows that the MC programs which model the parton cascade by implementing either CDM (ARIADNE[20], DJANGO) or PS (RAPGAP) describe the data. Additional gluon interactions in SCI (LEPTO)[19] which soften the spectra produced by PS is favoured by the data at high $x_{p}$ but disfavoured at low $x_{p}$. The string model of hadronisation (RAPGAP) provides a better description of the data than the cluster model (HERWIG).

\section{NEUTRAL STRANGE PARTICLES}

The inclusive production of the neutral strange hadrons $K_{s}^{0}, \Lambda, \bar{\Lambda}$ has been measured in ep with the ZEUS detector using the data accumulated 
A. Falkiewicz
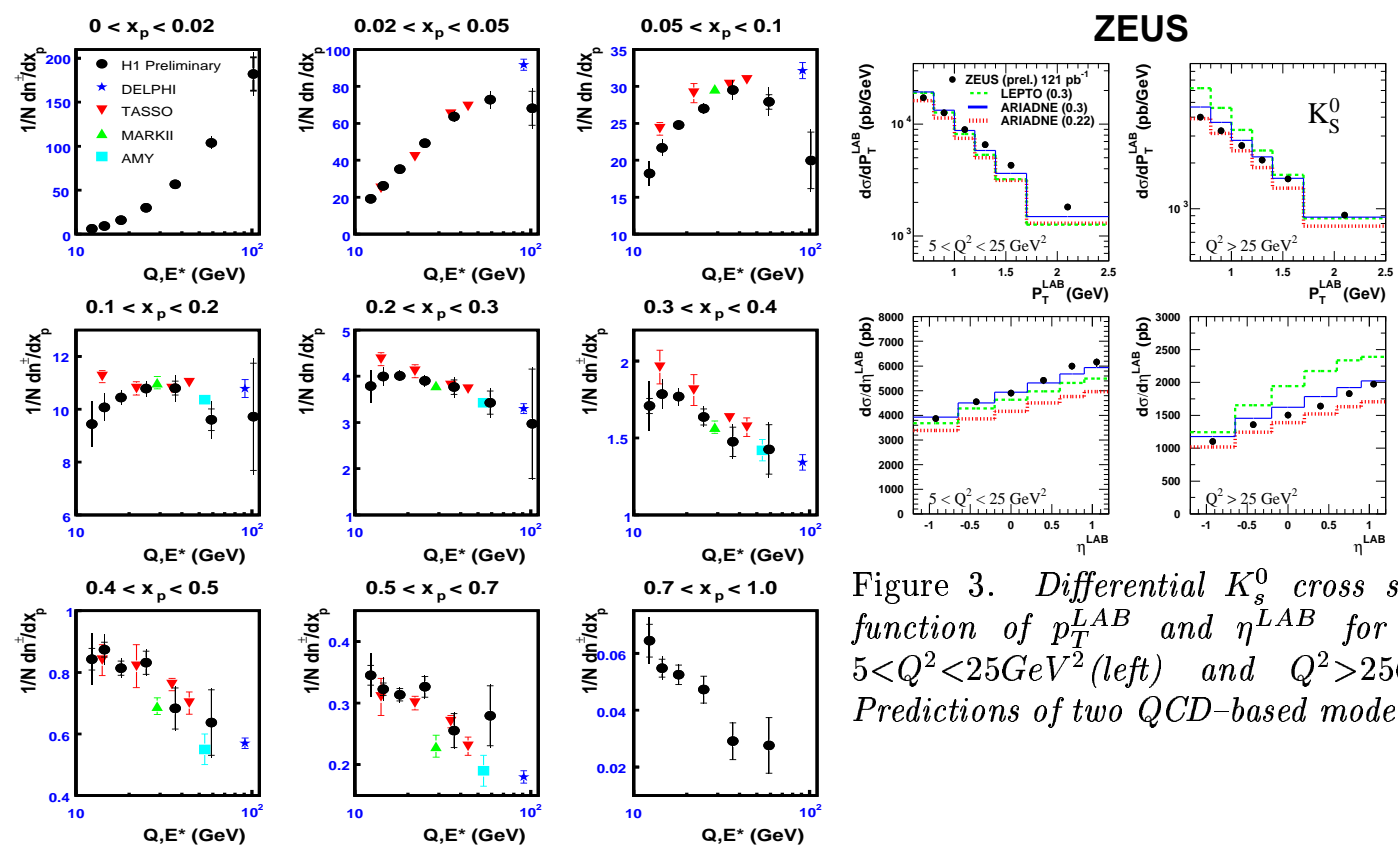

Figure 1. H1 data for the event normalised inclusive scaled momentum spectrum as a function of $Q$ for 9 different $x_{p}$ regions. Also shown are data from various experiments $e^{+} e^{-}\left(\right.$taking $\left.Q=E^{\star}\right)$
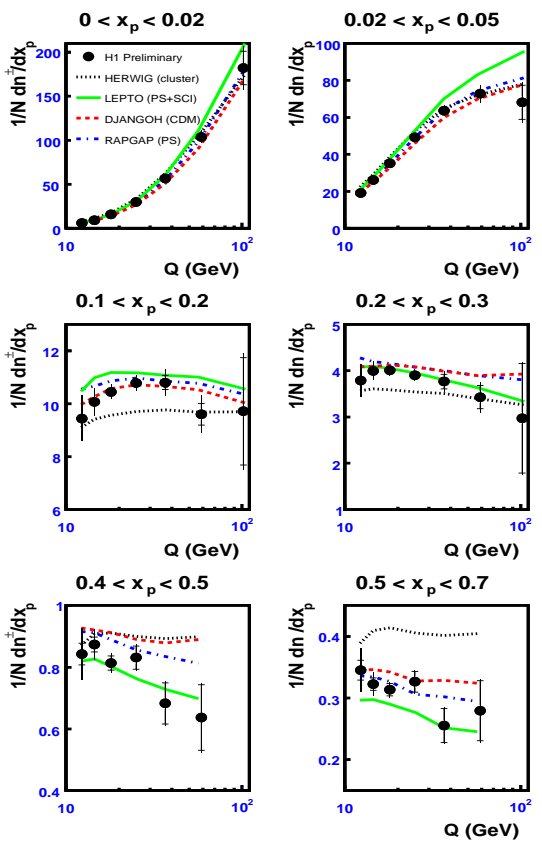

Figure 2. H1 data for the event normalised inclusive scaled momentum spectrum as a function of $Q$ for 9 different $x_{p}$ regions. Also shown are prdictions from different models of the hadronisation and fragmentation process implemented in Leading Order matrix element MC programs.
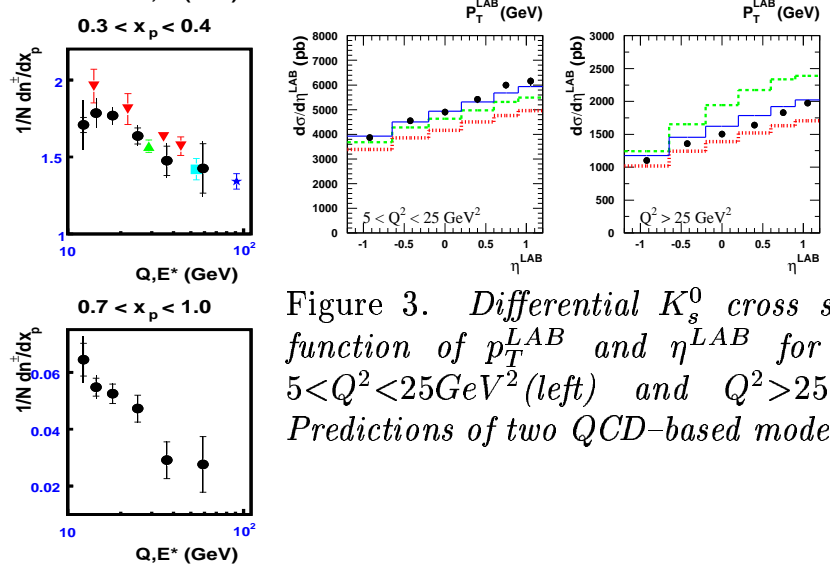

Figure 3. Differential $K_{s}^{0}$ cross sections as a function of $p_{T}^{L A B}$ and $\eta^{L A B}$ for events with $5<Q^{2}<25 \mathrm{GeV}^{2}$ (left) and $Q^{2}>25 \mathrm{GeV}^{2}$ (right). Predictions of two QCD-based models are shown.

\section{ZEUS}
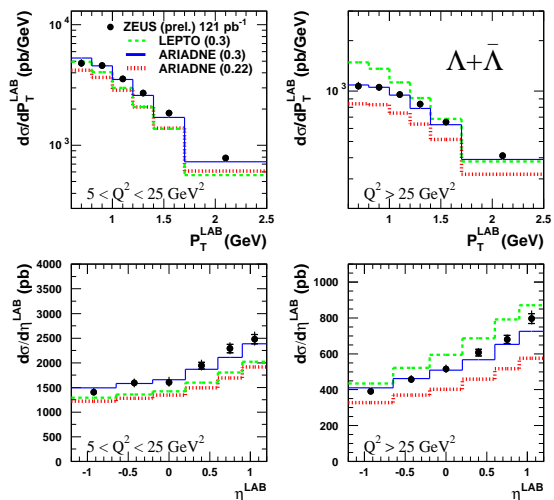

Figure 4. Differential $\Lambda+\bar{\Lambda}$ cross sections as a function of $p_{T}^{L A B}$ and $\eta^{L A B}$ for events with $5<Q^{2}<25 \mathrm{GeV}^{2}$ (left) and $Q^{2}>25 \mathrm{GeV}^{2}$ (right). Predictions of two QCD-based models are shown.

\section{ZEUS}
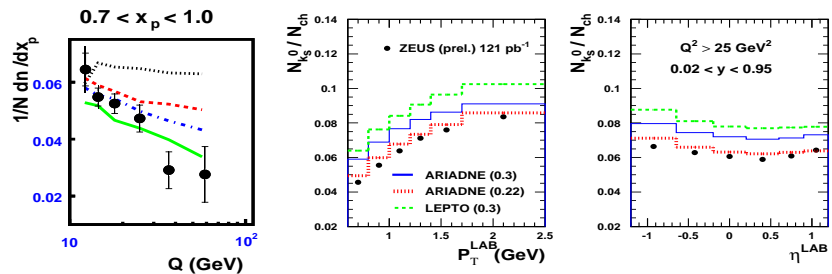

Figure 5. Strange to light hadron ratio as a function of $p_{T}^{L A B}$ and $\eta^{L A B}$ for events with $Q^{2}>25 \mathrm{GeV}^{2}$. 
from 1996 to 2000 correspondig to an integrated luminosity of $121 p b^{-1}[21]$. Cross sections, relative yields of strange and charged light hadrons have been measured in two regions of avaliable phasespace: at low $Q^{2}, 5<Q^{2}<25 \mathrm{GeV}^{2}$ and at high $Q^{2}$, $Q^{2}>25 \mathrm{GeV}^{2}$.

Figures 3 and 4 show differential cross sections for the $K_{s}^{0}$ and $\Lambda+\bar{\Lambda}$ production as a function of their transverse momentum $\left(p_{T}^{L A B}\right)$ and pseudorapidity $\left(\eta^{L A B}\right)^{3}$ in laboratory. The DIS cross sections are compared to prediction of ARIADNE and LEPTO MC. ARIADNE with strangeness suppresion factor $\lambda_{s}=0.3$ describes the data resonably well although for the high $Q^{2}$ data the $K_{s}^{0}$ cross section is overestimated.

Description of the data by ARIADNE with $\lambda_{s}=0.22$ is less satisfactory but in general, it looks as if varying one parameter is not sufficient to describe all cross sections well simultaneously. LEPTO does not describe the data well.

In Figure 5 strange to light charged hadron ratio is shown as a function of $p_{T}^{L A B}$ and $\eta^{L A B}$ for the high $Q^{2}$ sample. ARIADNE and LEPTO predictions are also shown. They follow the data resonably well preferring the $\lambda_{s}$ to be smaller than 0.3. $\lambda_{s}$ appears to be different for the description of inclusive cross sections and the ratio of strange to light charged hadrons.

\section{SEARCH FOR EXOTIC $\Theta^{+}, \Theta_{c}$ HADRONS}

\subsection{Strange pentaquark $\Theta^{+}$}

A search for a narrow baryonic resonance decaying to $K_{s}^{0} p$ or $K_{s}^{0} \bar{p}$ was made in DIS with the $\mathrm{H} 1$ and ZEUS detectors. The ZEUS results[22], based on $e p$ data taken in the years 1996-2000 with an integrated luminosity of $121 p b^{-1}$, support the existence of such a state in kinematic region at $Q^{2} \geq 20 \mathrm{GeV}^{2}$, with mass of $1521.5 \pm$ $1.5(\text { stat })_{-1.7}^{+2.8}($ syst. $) \mathrm{MeV}$ and the measured width of $\sigma=6.1 \pm 1.6(\text { stat })_{-1.4}^{+2.0}($ syst $) \mathrm{MeV}$. Figure 6 shows the signal with statistical significance $\sim 4.6 \sigma$ at high $Q^{2}$. The $\Theta^{+}$production cross section is

\footnotetext{
${ }^{3}$ The pseudorapidity $\eta^{L A B}$ is defined as $\eta^{L A B}=-$ lantan $(\theta / 2)$, with polar angle $\theta$ being measured with respect to positive $z$-axis, which is given by the proton beam.
}

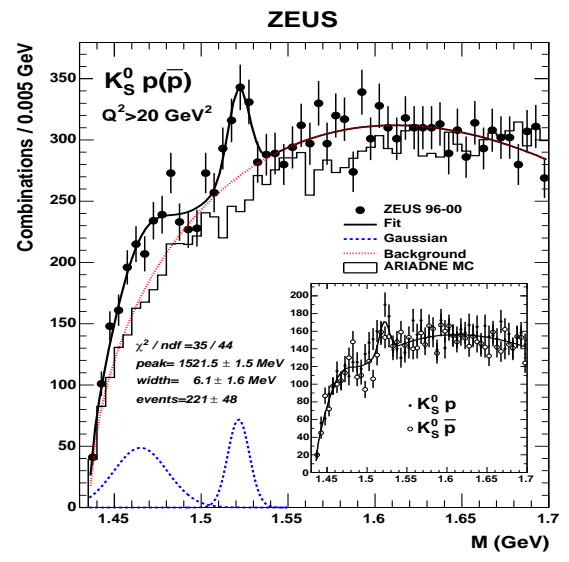

Figure $6 . K_{s}^{0} p$ or $K_{s}^{0} \bar{p}$ invariant mass spectrum for $Q^{2}>20 G e V^{2}$ The solid line is the result of a fit to the data using a three-parameter background function plus two Gaussians. The dashed lines show the Gaussian components and the dotted ones the background according to this fit. The histogram shows the prediction of ARIADNE MC normalised to the data in the mass region above $1650 \mathrm{MeV}$. The inset shows the $K_{s}^{0} \bar{p}$ (open circles) and $K_{s}^{0} p$ (black dots) separately, compared to the result of the fit to the combined sample scaled by a factor of 0.5 .

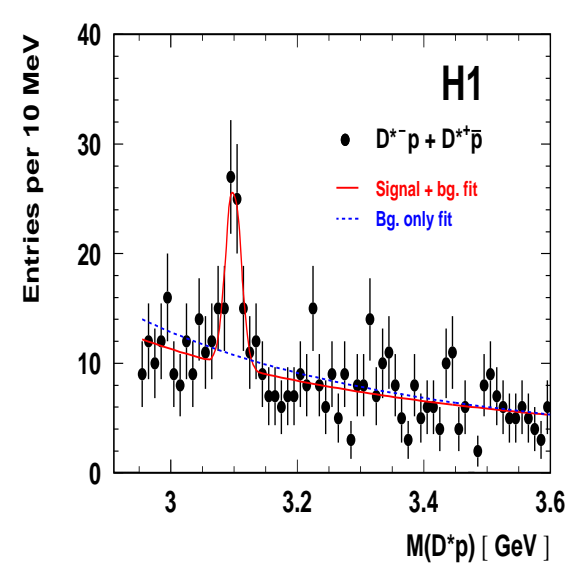

Figure 7. $D^{*-} p+D^{*+} \bar{p}$ invariant mass distribution compared with the results of a fit in which both signal and background components are inluded (solid line) and with the results of a fit in which only the background is included (dashed line) 
$125 \pm 27(\text { stat })_{-28}^{+36}($ syst $) p b$.

The $K_{s}^{0} p$ or $K_{s}^{0} \bar{p}$ invariant mass distributions obtained recently by H1 experiment[23] for 19962000 year data corresponding to an integrated luminosity of $74 p b^{-1}$, do not show any significant peak in mass range from threshold up to $1.7 \mathrm{GeV}$. At a $\Theta^{+}$mass of $1.52 \mathrm{GeV}$ an upper limit on the $\Theta^{+}$production cross section of $72 p b$ at the $95 \%$ confidence level is found. This upper limit is not in contradiction with ZEUS observed cross section.

\subsection{Charm pentaquark $\Theta_{c}$}

H1 and ZEUS experiments made a search for a narrow resonance decaying to $D^{*-} p$ and $D^{*+} \bar{p}$.

The H1 result [24] based on 1996-2000 year data with an integrated luminosity of $75 \mathrm{pb}^{-1}$ supports the existence of $\Theta_{c}$ state with a mass of $3099 \pm 3$ (stat) \pm 5 (syst. $) M e V$ and the measured width of $\sigma=12 \pm 3$ (stat) $M e V$. Figure 7 shows the signal with statistical significance $\sim 5.4 \sigma$. An acceptance corrected ratio of cross sections $R_{\text {cor }}\left(D^{*} p / D^{*}\right)=\left(1.59 \pm 0.33(\text { stat } .)_{-0.45}^{+0.33}(\right.$ syst. $\left.)\right) \%$ for the visible $D^{*} p$ and $D^{*}$ range has been set [25].

No resonance structure was observed in the $D^{*-} p$ and $D^{*+} \bar{p}$ invariant mass distribution obtained recently by ZEUS experiment[26] using data taken in the years 1995-2000 with an integrated luminosity of $126 \mathrm{pb}^{-1}$. An upper limit on the acceptance corrected ratio $R_{c o r}\left(D^{*} p / D^{*}\right)$ of $0.59 \%$ at the $95 \%$ confidence level is found. The ZEUS result is not compatible with the $\mathrm{H} 1$.

\section{CONCLUSIONS}

The recent results from $\mathrm{H} 1$ and ZEUS on production of neutral strange particles $\left(K_{s}^{0}, \Lambda, \bar{\Lambda}\right)$, charged and exotic $\left(\Theta^{+}, \Theta_{c}\right)$ hadrons in DIS ep collisions at HERA have been presented.

The cross section normalised scaled momentum distribution of charged final state hadrons measured at high $Q^{2}$ in the Breit frame support the concept of quark fragmentation universality in $e p$ collisions and $e^{+} e^{-}$annihilation as implemented in MC models with either PS or CDM. The string model of hadronisation provides a better description of the data than the cluster model as imple- mented in HERWIG.

Inclusive $\Lambda$ and $K_{s}^{0}$ cross sections are best described by ARIADNE with $\lambda_{s}=0.3$. The relative yields of strange and charged light hadrons is also described by ARIADNE but at lower $\lambda_{s}=0.2$.

Both $\mathrm{H} 1$ and ZEUS experiments performed searches for exotic baryons. ZEUS has found evidence for the production of the strange pentaquark $\Theta^{+}$. This observation has not been confirmed by the $\mathrm{H} 1$ collaboration.

$\mathrm{H} 1$ has found evidence for the existence of a narrow charmed baryon decaying to $D^{*} p$. This result has not been confirmed by the ZEUS collaboration.

\section{REFERENCES}

1. H1 collab., C. Adloff et al., ICHEP2006: 33rd Int. Conf. on HEP, $26.07-2.08 .06$.

2. H1 collab., S. Aid et al., Nucl. Phys. B445 (1995) 3.

3. H1 collab., C. Adloff et al., Nucl. Phys. B504 (1997) 3.

4. H1 collab., C. Adloff et al., ICHEP98: 29th Int. Conf. on HEP (Vancover, Canada), July 1998 p531.

5. ZEUS Collab., M. Derrik et al., Z. Phys. C67 (1995) 93.

6. ZEUS Collab., M. Derrik et al., Phys. Lett.B414 (1997) 428.

7. ZEUS Collab., J. Breitweg et al., Eur. Phys. J. C11 (1999) 251.

8. M. Bengtsson and T. Sjöstrand, Z. Phys. C37 (1988) 465.

9. G. Gustafson, Phys. Lett. B175 (1986) 453.

10. B. Andersson et al., Phys. Rep. 97 (1983) 31.

11. B. R. Webber, Nucl. Phys. B238 (1984) 492.

12. H. Jung, Comp. Phys. Comm. 86 (1995) 147.

13. A. Kwiatkowski, H. Speisberger and H. J. Mohring, Comp. Phys. Comm. 69 (1992) 155.

14. G. Marchesini et al., Comp. Phys. Comm. 67 (1992) 465.

15. TASSO Collab., W. Braunschweig et al., Z. Phys. C47 (1990) 187.

16. MARK II Collab., A Petersen et al., Phys. Rev. D37 (1988) 1.

17. AMY Collab., Y. K. Li et al., Phys. Rev D41 (1990) 2675. 
18. DELPHI Collab., P. Abreu et al., Phys. Lett. B311 (1993) 408.

19. A. Edin, G. Ingelman and J. Rathsman, Phys.Lett. B366 (1996) 371.

20. L. Lönnblad, Comp. Phys. Comm. 71 (1992) 15.

21. ZEUS Collab., S. Chekanov et al., ICHEP2006: 33rd Int. Conf. on HEP, 26.07 - 2.08.06.

22. ZEUS Collab., S. Chekanov et al., Phys. Lett. B 591 (2004) 7.

23. H1 Collab., A. Aktas et al., Phys. Lett. B 639 (2006) 202.

24. H1 Collab., A. Aktas et al., Phys. Lett. B588 (2004) 17.

25. H1 Collab., A. Aktas et al., XXII Int. Symp. on Lepton-Photon Interac., 30.07.05.

26. ZEUS Collab., S. Chekanov et al., Eur. Phys. Jour. C 38 (2004) 29. 